[Article]

www.whxb.pku.edu.cn

\title{
超疏水表面微纳二级结构对冷凝液滴最终状态的影响
}

\author{
刘天庆* 孙玮 孙相或 艾宏儒 \\ (大连理工大学化工学院, 辽宁大连 116024)
}

\begin{abstract}
摘要: 从超疏水表面 $(\mathrm{SHS})$ 上初始冷凝液核长大、合并、形成初始液斑开始, 分析计算了冷凝液斑变形成为 Wenzel 或 Cassie 液滴过程中界面能量的变化, 并以界面能曲线降低、是否取最小值为判据, 确定冷凝液滴的最 终稳定状态. 计算结果表明: 在只有微米尺度的粗精结构表面上, 冷凝液滴的界面能曲线一般都是先降低再升 高, 呈现 Wenzel 状态; 而当表面具有微纳米二级粗精结构, 且纳米结构的表面空气面积分率较高时, 冷凝液滴的 能量曲线持续降低, 直至界面能最小的 Cassie 状态, 因此可以自发地形成 Cassie 液滴. 还计算了文献中具有不 同结构参数的 SHS 上冷凝液滴的状态和接触角, 并与实验结果进行了比较, 结果表明, 计算的冷凝液滴状态与 实验观察结果完全吻合. 因此, 微纳二级结构是保持冷凝液滴在 SHS 上呈现 Cassie 状态的重要因素.
\end{abstract}

关键词：超疏水表面；微纳结构；表面；界面；自由能；冷凝

中图分类号: 0647

\section{Effect of Hierarchical Architecture of Super-Hydrophobic Surface on the Condensed Drop's Final State}

\author{
LIU Tian-Qing * SUN Wei SUN Xiang-Yu AI Hong-Ru \\ (School of Chemical Engineering, Dalian University of Technology, Dalian 116024, Liaoning Province, P. R. China)
}

\begin{abstract}
The interface free energy of a local condensate from the growth and combination of numerous initial condensation nuclei was calculated during its shape changes from an early flat shape to a Wenzel or Cassie state on the super-hydrophobic surface (SHS). The final state of the condensed drop was determined according to whether the interface free energy continuously decreased or it had a minimum value. Our calculations indicate that condensation drops on a surface only with micro roughness display Wenzel state because the interface free energy curve of a condensed drop first decreases and then increases, existing a minimum value corresponding to Wenzel drop. On a surface with appropriate hierarchical roughness, however, the interface energy curve of a condensed drop will constantly decline until it reaches the Cassie state. Therefore, a condensed drop on a hierarchical roughness surface can spontaneously reach the Cassie state. In addition, the states and apparent contact angles of condensed drops on a SHS with different structural parameters were calculated and compared with experimental observations. Results show that the calculated condensed drop states agree well with the experimental results. It can be concluded that micro and nano hierarchical roughness is the key structural factor responsible for sustaining condensed drops in the Cassie state on a SHS.
\end{abstract}

Key Words : Super-hydrophobic surface; Micro and nano hierarchical structure; Surface; Interface; Free energy; Condensation

\footnotetext{
Received: April 29, 2010; Revised: July 6, 2010; Published on Web: September 13, 2010.

"Corresponding author. Email: liutq@dlut.edu.cn; Tel: +86-4111-84706360.

The project was supported by the National Natural Science Foundation of China (50876015).

国家自然科学基金(50876015)资助项目

(C) Editorial office of Acta Physico-Chimica Sinica
} 
滴状冷凝具有很高的传热系数, 可比常见的膜 状冷凝传热系数高几十倍. 冷凝传热在石油化工和 发电等工业生产中以及空调和制冷等过程中被大量 采用, 如果能在这些过程中均实现滴状冷凝, 则必定 会大大减少换热设备的面积与尺寸, 降低能源消耗, 从而带来显著的社会和经济效益.

滴落在超疏水表面(SHS)上的液滴可以呈现表 观接触角大于 $150^{\circ}$, 且滚动角很小的 Cassie 形态. 这很容易使我们联想到蒸气会在SHS 上形成良好 的滴状冷凝, 即 SHS 应该能成为实现滴状冷凝的理 想表面.

然而现有为数不多的 SHS 上冷凝实验结果却 表明, 在只有微米粗粘结构的表面上所形成的冷凝 液滴往往失去超疏水性 ${ }^{[1]}$, 液滴一般呈现 Wenzel 状 态 ${ }^{[2-5]}$ 或 Wenzel 与 Cassie 的混合状态 ${ }^{[6-7]}$, 滴状冷凝 传热效果不理想 ${ }^{[0-9]}$. 而当表面具有微纳米尺度二级 结构或只有纳米粗糙结构时, 冷凝液滴就能够呈现 Cassie 状态 ${ }^{[10-12]}$, 并易于滚动脱落.

冷凝液滴为什么只有在具有微纳二级结构或纳 米粗䊁结构的表面才能保持超疏水状态? 冷凝过程 中最初的微小液滴合并后经过怎样的演变过程最终 形成稳定的冷凝液滴? 冷凝液滴的最终状态与 SHS 的微纳粗粘结构参数的定量关系是什么? 所有这些 问题都尚未明确, 需要从理论上进行分析. 为此, 本 文从液滴界面能量计算人手, 对冷凝液滴在其形态 改变时的界面能量变化曲线进行计算, 按液滴能量 减小并直至达到极小值为判据, 确定各种粗鋉结构 的 SHS 上冷凝液滴的最终稳定状态.

在液滴表面自由能计算方面, 已经有若干研究 者针对滴落在 SHS 上尺度较大的液滴进行了研究, 分析了液滴的表观接触角和接触角滞后 ${ }^{[13-17]}$ 、液滴

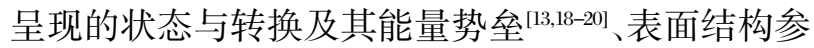
数的影响 ${ }^{[13-21]}$ 等问题. 但所有这些研究均不是针对 冷凝液滴进行的, 而事实是冷凝液滴在 SHS 上的形 态和行为与滴落的液滴行为差异很大, SHS 上冷凝 形成的液滴行为与其表面自由能的关系尚未见文献 报道.

\section{1 理论推导}

具有微纳二级结构表面上液滴表观接触角的计 算公式推导如下. 以图 1 所示的长方体微纳米粗糙 结构为例, 首先定义以下参数,

Cassie 粗粗系数:

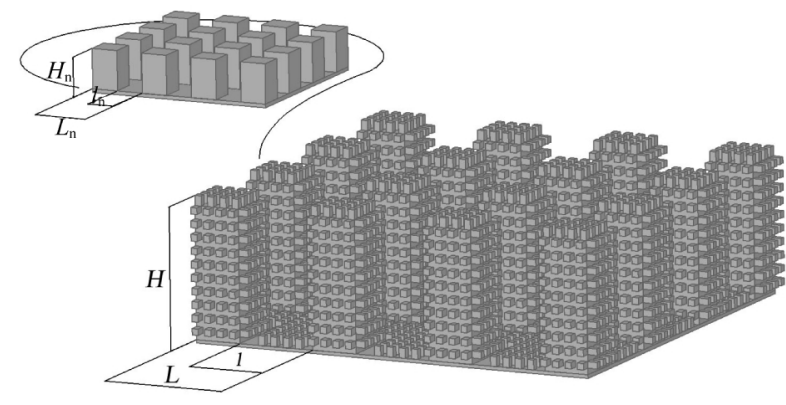

图 1 微纳二级结构超疏水表面示意图

Fig.1 Schematic of SHS with micro nano hierarchical textured pillars

SHS: super-hydrophobic surface

微米级: $f=\left(1-\frac{l}{L}\right)^{2}$

纳米级: $f_{\mathrm{n}}=\left(1-\frac{l_{\mathrm{n}}}{L_{\mathrm{n}}}\right)^{2}$

Wenzel 粗楉系数,

微米级: $r=1+4 H \frac{f}{L-l}$

纳米级: $r_{\mathrm{n}}=1+4 H_{\mathrm{n}} \frac{f_{\mathrm{n}}}{L_{\mathrm{n}}-l_{\mathrm{n}}}$

式中 $f 、 f_{\mathrm{n}}$ 分别是 SHS 微、纳米结构凸起肋固体所占 的投影面积分率; $r 、 r_{\mathrm{n}}$ 分别是微、纳米结构实际面积 与投影面积之比; $l 、 l_{\mathrm{n}}$ 分别为微、纳米结构凸起胁间 空隙宽度; $L 、 L_{\mathrm{n}}$ 分别为微、纳米结构凸起胁间距; $H$ 、 $H_{\mathrm{n}}$ 分别是微、纳米结构凸起肋高度.

(1) Cassie 或 Cassie 与 Wenzel 中间状态的液滴 如图 2(a)所示, 具有微纳二级结构 SHS 上的液 滴根部与微米凸起胁上部及部分侧面上的纳米尺度 凸起固相相接触, 其余部分均与气体接触. 按照分析 微米尺度上液滴界面能 ${ }^{[13]}$ 相似的方法, 在现在的微 纳二级结构表面上选定不变投影面积 $A_{\text {total }}$, 则 $A_{\text {total }}$ 内包括某个液滴的总界面能为

$$
E_{\text {surf }}=E_{\text {ext }}+E_{\text {base }}+E_{\left(A_{\text {todal }}-A_{\text {bax }}\right)}
$$

式中 $E_{\text {sur }}$ 是选定系统的总界面能, $E_{\text {ext }}$ 为液滴上方外 表面的气-液界面能, $E_{\mathrm{base}}$ 是液滴底部所包括的各种

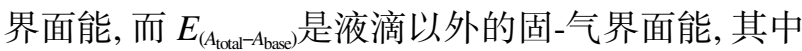
液滴上方外表面的气液界面能为

$$
E_{\text {ex }}=A_{\text {ex }} \sigma_{\mathrm{lg}}
$$

其中 $A_{\mathrm{ext}}$ 是液滴上部的外表面积, $\sigma_{\mathrm{lg}}$ 为气-液界面张 力. 液滴底部所包括的各种界面能为

$$
\begin{aligned}
E_{\text {base }}= & A_{\text {base }}(1-f) \sigma_{\mathrm{lg}}+A_{\text {base }} f\left(1+\frac{4 h}{L-l}\right)\left[f_{\mathrm{n}} \sigma_{\mathrm{sl}}+\left(1-f_{\mathrm{n}}\right) \sigma_{\mathrm{lg}}+\right. \\
& \left.\left(1-f_{\mathrm{n}}+r_{\mathrm{n}}-1\right) \sigma_{\mathrm{sg}}\right]+A_{\text {base }}\left[1-f+\frac{4(H-h) f}{L-l}\right] r_{\mathrm{n}} \sigma_{\mathrm{sg}} \\
& (0 \leqslant h<H)
\end{aligned}
$$


其中 $A_{\text {base }}$ 是液滴底部的投影面积, $\sigma_{\mathrm{sl}} 、 \sigma_{\mathrm{sg}}$ 分别为固液和固-气界面张力, $h$ 是液滴嵌人 SHS 微米结构凸 起肋间的深度. 液滴以外的固气界面能为

$$
E_{\left(A_{\text {total }}-A_{\text {base }}\right.}=\left(A_{\text {total }}-A_{\text {base }}\right) r r_{\mathrm{n}} \sigma_{\text {sg }}
$$

整理以上各种界面能后可以得到总界面能为

$$
E_{\text {surf }}=A_{\text {exx }} \sigma_{\mathrm{lg}}+A_{\text {base }}(1-f) \sigma_{\mathrm{lg}}-A_{\text {base }} f\left(1+\frac{4 h}{L-l}\right) \cos \theta_{\mathrm{n}} \sigma_{\mathrm{lg}}+
$$

$$
A_{\text {total }} r r_{\mathrm{n}} \sigma_{\mathrm{sg}}
$$

其中

$$
\begin{aligned}
& \cos \theta_{\mathrm{n}}=f_{\mathrm{n}} \cos \theta_{0}+f_{\mathrm{n}}-1 \\
& \cos \theta_{0}=\frac{\sigma_{\mathrm{sg}}-\sigma_{\mathrm{sl}}}{\sigma_{\mathrm{lg}}}
\end{aligned}
$$

式中 $\theta_{\mathrm{n}}$ 为液滴在纳米表面上的表观接触角, 而 $\theta_{0}$ 是 其在光滑表面的本征接触角. 式(5)可以整理成

$$
E_{\text {surf }}=A_{\text {ex }} \sigma_{\mathrm{lg}}+C_{\text {comp }} A_{\text {base }}+A_{\text {total }} r r_{\mathrm{n}} \sigma_{\mathrm{sg}}
$$

其中非润湿状态系数 $C_{\text {comp }}$ 为

$$
C_{\text {comp }}=-\sigma_{\mathrm{lg}}\left[f\left(1+\frac{4 h}{L-l}\right) \cos \theta_{\mathrm{n}}+f-1\right]
$$

再将液滴外表面积和底面积表达成液滴体积 $V$ 与 表观接触角 $\theta$ 的关系:

$$
\begin{aligned}
& A_{\text {ex }}=2 \pi\left[\left(\frac{3 V}{\pi}\right)^{2 / 3} \frac{1-\cos \theta}{\left(2-3 \cos \theta+\cos ^{3} \theta\right)^{2 / 3}}\right] \\
& A_{\text {base }}=\pi\left[\frac{3 V}{\pi} \frac{1}{\left(2-3 \cos \theta+\cos ^{3} \theta\right)}\right]^{2 / 3} \sin ^{2} \theta
\end{aligned}
$$

代人界面能表达式(6):

$$
\begin{aligned}
E_{\text {surf }}= & \pi\left(\frac{3 V}{\pi}\right)^{2 / 3} \frac{1}{\left(2-3 \cos \theta+\cos ^{3} \theta\right)^{2 / 3}} \cdot \\
& {\left[2 \sigma_{\mathrm{lg}}(1-\cos \theta)+C_{\text {comp }} \sin ^{2} \theta\right]+\sigma_{\text {sg }} A_{\text {total }} r r_{\mathrm{n}} }
\end{aligned}
$$

对 $\cos \theta$ 求导并取极值可以获得对应的液滴能 量最小时的表观接触角为

$$
\cos \theta=f\left(1+\frac{4 h}{L-l}\right) \cos \theta_{\mathrm{n}}+f-1
$$

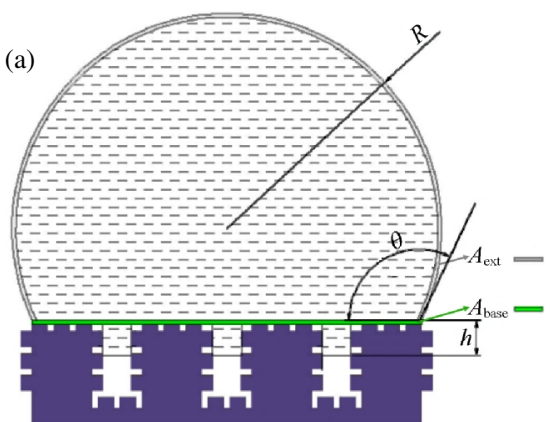

可见, 只需将只有微米结构的 Cassie 或 Cassie 与 Wenzel 的中间状态方程中的 $\theta_{0}$ 一项换成纳米表 面的表观接触角 $\theta_{\mathrm{n}}$, 就成为微纳二级结构时的表观 接触角方程.

(2) Wenzel 状态的液滴

如图 2(b) 所示, 液滴根部与全部微米尺度的肋 表面接触, 在纳米尺度上液体只与纳米凸起肋的上 部固相相接触, 而不能进人纳米凸起肋的内部, 即液 滴在纳米粗粘结构上永远呈 Cassie 状态. 此时液滴底部所包括的各种界面能为

$$
\begin{aligned}
E_{\text {base }}= & A_{\text {base }} r\left[f_{\mathrm{n}} \sigma_{\mathrm{sl}}+\left(1-f_{\mathrm{n}}\right) \sigma_{\mathrm{lg}}+\left(1-f_{\mathrm{n}}+r_{\mathrm{n}}-1\right) \sigma_{\mathrm{sg}}\right]+ \\
& \left(A_{\text {total }}-A_{\text {base }}\right) r r_{\mathrm{n}} \sigma_{\mathrm{sg}}
\end{aligned}
$$

或者:

$$
E_{\text {base }}=\sigma_{\mathrm{lg}} A_{\text {base }} r\left[f_{\mathrm{n}} \frac{\sigma_{\mathrm{sl}}-\sigma_{\mathrm{sg}}}{\sigma_{\mathrm{lg}}}+1-f_{\mathrm{n}}\right]+A_{\text {total }} r r_{\mathrm{n}} \sigma_{\mathrm{sg}}
$$

液滴上方外表面和液滴以外固气界面的界面能同 上. 于是, 整理各种界面能后可以得到总界面能为

$$
E_{\text {surf }}=A_{\text {ex }} \sigma_{\text {lg }}+C_{\text {Wenze }} A_{\text {base }}+A_{\text {total }} r r_{\mathrm{n}} \sigma_{\text {sg }}
$$

其中润湿状态系数 $C_{\text {Wenzel }}$ 为

$$
C_{\text {Wenzel }}=-\sigma_{\mathrm{lg}} r \cos \theta_{\mathrm{n}}
$$

类似前面的分析, 将液滴外表面积和底面积表 达成液滴体积与接触角的关系并代人界面能表达式 中, 并对 $\cos \theta$ 求导和取极值可以获得对应的液滴能 量最小时的表观接触角为

$$
\cos \theta=r \cos \theta_{\mathrm{n}}
$$

可见, 只需将只有微米结构的 Wenzel 方程中 的 $\theta_{0}$ 一项换成纳米表面的表观接触角 $\theta_{\mathrm{n}}$, 就成为具 有微纳二级结构时的 Wenzel 表观接触角方程.

\section{2 超疏水表面冷凝液滴变形过程的能量分析}

SHS 上冷凝液滴的形成过程如图 3 所示. 初始 形成的纳米尺度微小液滴长大到液滴临界尺度后开

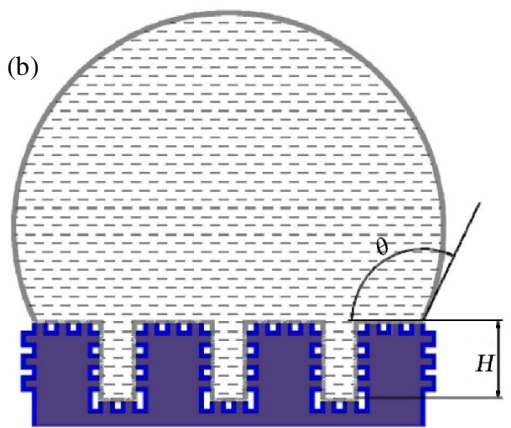

图 2 微纳二级结构表面上的非润湿和润湿液滴示意图

Fig.2 Schematic of a composite or wetted drop on SHS with micro nano hierarchical textured pillars 
始合并, 众多小液滴合并的结果将在 SHS 部分区域 填满微米结构的凹陷处, 形成上部较为平缓的冷凝 液滴斑 ${ }^{[1-2]}$. 冷凝液的这种状态能量较高, 将会自发 的向 Wenzel 或 Cassie 状态变化. 但是具体向哪种 状态变化, 需要根据液滴能量的计算进行判断, 即液 滴形态将向其能量减小的方向改变, 并当液滴能量 降低至极值时即为液滴的最终稳定状态.

图 3(d)所示的初期冷凝液斑向 Wenzel 和Cassie 状态变化可以通过不断减小液滴底半径的方式进 行, 如果液滴底半径减小到某一数值时其能量到达 极小值, 这时液滴所呈现的 Wenzel 状态就是冷凝 液滴的最终状态; 如果在液滴半径减小的过程中其 能量持续降低, 直到 Cassie 状态, 那么液滴的最终 状态就是 Cassie 状态.

初期冷凝液斑或 Wenzel 液滴向 Cassie 状态转 变的另一途径是液滴底半径不变、但液滴根部离开 超疏水微米结构的底部并向上移动. 但是文献 ${ }^{[20]}$ 及 本文的计算结果均表明, 液滴根部在离开表面底部 时其能量会突然升高, 形成能垒, 不会自发地通过这 种途径变成 Cassie 状态. 因此本文以下主要计算了 液滴底半径减小的过程中其能量的变化.

不同状态时冷凝液滴能量的计算公式 Cassie

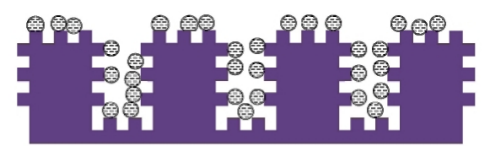

(a)

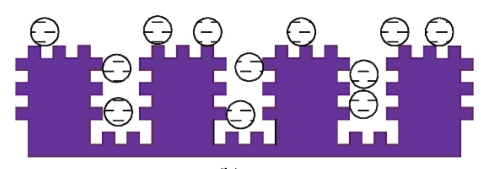

(b)

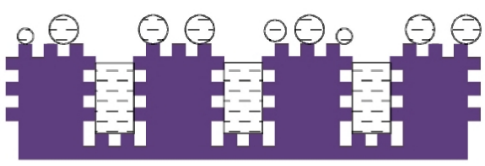

(c)

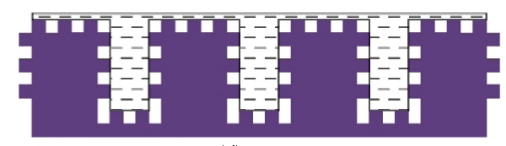

(d)
状态为式(6), Wenzel 状态为式(14), 冷凝液斑尚未 填满微米凸起肋空间时:

$$
\begin{aligned}
E_{\text {surf }}= & A_{\text {base }}\left\{[ 1 - f + \frac { 4 ( H - h ) f } { L - l } ] \left[f_{\mathrm{n}} \sigma_{\mathrm{sl}}+\left(1-f_{\mathrm{n}}\right) \sigma_{\mathrm{lg}}+\right.\right. \\
& \left.\left(1-f_{\mathrm{n}}+r_{\mathrm{n}}-1\right) \sigma_{\mathrm{sg}}\right]+(1-f) \sigma_{\mathrm{lg}}+f\left(1+\frac{4 h}{L-l}\right) r_{\mathrm{n}} \sigma_{\mathrm{sg}}- \\
& \left.r r_{\mathrm{n}} \sigma_{\mathrm{sg}}\right\}+A_{\text {total }} r r_{\mathrm{n}} \sigma_{\mathrm{sg}}=A_{\text {base }}\{1-f-[(1-f)+ \\
& \left.\left.\frac{4(H-h) f}{L-l}\right] \cos \theta_{\mathrm{n}}\right\} \sigma_{\mathrm{lg}}+A_{\text {total }} r r_{\mathrm{n}} \sigma_{\mathrm{sg}}
\end{aligned}
$$

式中 $4 /(L-l)$ 的意义为微米凸起胁的周长与其截面积 之比, 只需将其它几何形状凸起胁的 $f 、 r$ 、周长和截 面积比等参数代人, 就可以获得其它几何形状 SHS 液滴相关界面能的计算公式.

SHS 不同的微纳二级结构参数下冷凝液滴典 型的能量变化曲线如图 4 所示 (以液斑初始状态为 基准计算自由能差). 本文是针对初期冷凝液斑只占 满 80\%微米肋沟的条件下做的计算, 因此, 在液滴 底半径减小、液位上升的过程中其能量一开始在不 断减小, 但当液滴上表面漫过凸起肋上表面时, 其能 量会有不同程度的突然增加. 然而, 对于实际的冷凝 过程, 液斑并不需要克服这个能垒, 因为冷凝在不断 发生, 即使初始液斑没有占满肋沟, 也会由于冷凝液

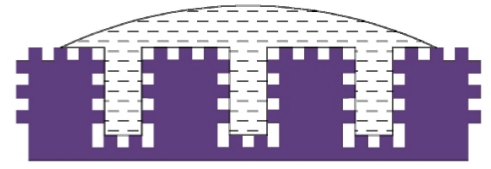

(e)

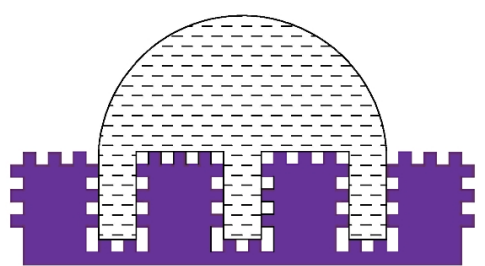

(f)

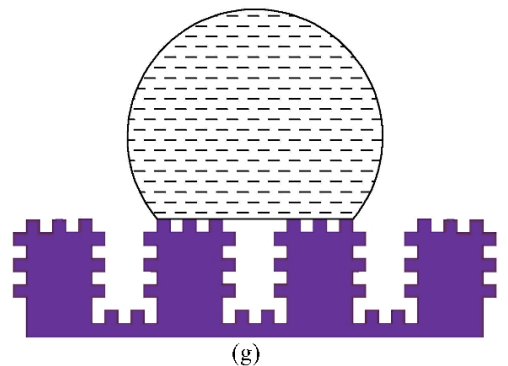

(g)

图 3 超疏水表面上冷凝液滴形成过程示意图

Fig.3 Schematic of formation of a condensed drop on SHS

(a) initial nuclei; (b) grown up and combined small drops; (c) liquid spot within pillar gaps formed by condensate; (d) liquid spot with flat external surface; (e) the condensate begins reducing its base radius; (f) Wenzel state drop; (g) Cassie state drop 
的不断形成而填满整个凸起肋. 因此我们只需要考 虑在液斑填满整个凸起肋高度以后液滴的形态和能 量的变化.

从图 4 可以看出, 在冷凝液斑充满凸起肋以后, 液滴的能量就开始不断下降, 在很多参数条件下, 液 滴能量会在某一液滴底半径时不再下降, 而在某些 参数条件下, 液滴能量会持续下降, 直至底半径为 0 的 Cassie 状态. 总体上看, SHS 的微纳米结构对液 滴能量有明显影响, 微米凸起肋之间的距离越小, 液 滴能量变化越明显, 液滴越容易形成 Cassie 状态; 而随着纳米凸起胁间距的增大, 液滴能量在减小, 液 滴也更容易转变成 Cassie 状态.

其它结构参数和液滴体积时的冷凝液滴能量变 化曲线规律类似.

\section{3 超疏水表面冷凝液滴最终状态的分析}

根据以上液滴在不同状态时的能量曲线, 可以 确定液滴最终的稳定状态: 在液滴底半径减少的过 程中如果其能量持续降低, 或者能量曲线的斜率一 直大于 0 , 则该液滴最终呈 Cassie 状态; 否则, 在能 量曲线的斜率出现 0 值时所对应的液滴为 Wenzel 状态. 在确定了液滴的最终状态的同时, 其接触角等 参数也同时获得.

按照以上方法, 本文对具有不同微纳尺度结构 参数的疏水表面上冷凝液滴的最终状态和相应的 接触角进行了计算, 结果分别如图 5 的(A1)-(A3)和 (B1)-(B3)所示. 可见, 当 $l_{\mathrm{n}}$ 为 0 或很小时, 即疏水表 面没有纳米二级结构时, 冷凝液滴需要在较小的 $l / L$ 或较大的 $H$ 值条件下才能成为 Cassie 状态, 并且这 时的接触角均小于 $150^{\circ}$, 因此液滴虽然呈 Cassie 状 态, 但并非属超疏水液滴. 随着 $l_{\mathrm{n}}$ 的增加, 即疏水表 面的纳米二级结构越来越明显时, 冷凝液滴更容易 呈 Cassie 状态, 并且相应的接触角在不断增加. 为 了表达冷凝液滴呈 Cassie 并且为超疏水状态, 本文 又计算了能同时满足 Cassie 条件和接触角大于等 于 $150^{\circ}$ 时所需的表面粗糙结构参数, 如图 5 的 $(\mathrm{Cl})-$ (C3)所示. 因此, 当表面具有明显的微纳二级粗䊁结 构时, 冷凝液滴容易成为超疏水状态的Cassie 液滴. 而当没有纳米结构时, 只有当 $H$ 很大、适宜的 $l / L$ 取 值范围之内的条件下, 冷凝液滴才能呈现超疏水的 Cassie 状态.

从图 5 还可以发现另一项有趣的计算结果, 出 现在 $l / L$ 较大的情况下, 此时无论表面是否具有纳
米二级结构, 冷凝液滴都呈现 Wenzel 状态, 并且接 触角较小, 特别是在 $l_{\mathrm{n}} / L_{\mathrm{n}}$ 也较小的条件下, 表观接 触角可以小到低于本征接触角的程度. 众所周知, 在 本征接触角大于 $90^{\circ}$ 的条件下, 粗粘表面上 Wenzel 液滴的表观接触角按照 Wenzel 方程不可能小于本 征接触角, 但是本计算却揭示冷凝所形成的液滴其 最终状态有可能完全不符合 Wenzel 公式, 这时的 液滴接触角低于 Wenzel 方程所确定的数值. 原因 在于在冷凝液滴的尺度下 $(100 \mu \mathrm{m}$ 左右 $)$, 超疏水微 米粗糙结构内的水体积相对于整个液滴体积已经不 可忽略, 因此, Wenzel 方程不再适用于尺度很小的 冷凝液滴.

本文对文献报道的 SHS 上的冷凝液滴状态和 接触角进行了计算并与实验结果进行比较, 如表 1 所示. 可见本文所计算的冷凝液滴形态与所有实验 结果都吻合. 首先仅有的三篇具有微纳二级结构或 只有纳米结构的表面上的冷凝实验都表明 ${ }^{[10-12]}$, 微 纳米结构表面上冷凝形成的液滴能呈现易于滚落的 Cassie 液滴, 而本计算也表明, 表面具有适宜微纳米 二级结构时, 冷凝液滴易于形成接触角大于 $150^{\circ}$ 的 Cassie 状态. 此外, 文献[6-7]均观察到同一表面上 可以形成 Wenzel 和 Cassie 两种液滴或混合型液滴. 本计算结果表明在所观察的冷凝液滴尺度范围内, 较小的液滴呈现 Wenzel 状态, 而相对较大的液滴 则可能呈现 Cassie 状态, 于是表面上确有可能形成 两种形态的液滴. 对于文献[1-3]的表面结构参数, 则对各种液滴体积其形态均为 Wenzel 状态, 计算 结果也与实测情况完全符合.

关于接触角, 有两篇文献的实测结果与计算结 果不符合. 其中文献[7]对于较大液滴(体积在 $\mu \mathrm{L}$ ) 的 实测接触角远低于本文的计算结果或 Wenzel 公式. 在液滴尺度较大时, 液滴根部在凸起肋间的液体体 积可以忽略, 此时液滴的接触角应该符合 Wenzel 方程, 并且当表面材料的本征接触角大于 $90^{\circ}$ 时, 表 观接触角不可能低于本征接触角. 文献[7]给出的本 征接触角为 $117.3^{\circ}$, 但是其所测量的表观接触角都 小于该数值, 这是难以解释的. 除非冷凝实验过程中 表面上的疏水涂层被破坏, 而该文献并没有说明冷 凝实验后其表面的本征接触角是否改变. 另一方面, 文献[3]所报道的接触角都明显高于本计算值或 Wenzel 公式对应的接触角. 经过仔细观察该文献的 冷凝液滴照片, 我们发现他们所形成的冷凝液滴底 部半径都非常小, 所覆盖的凸起肋个数只有几个, 而 

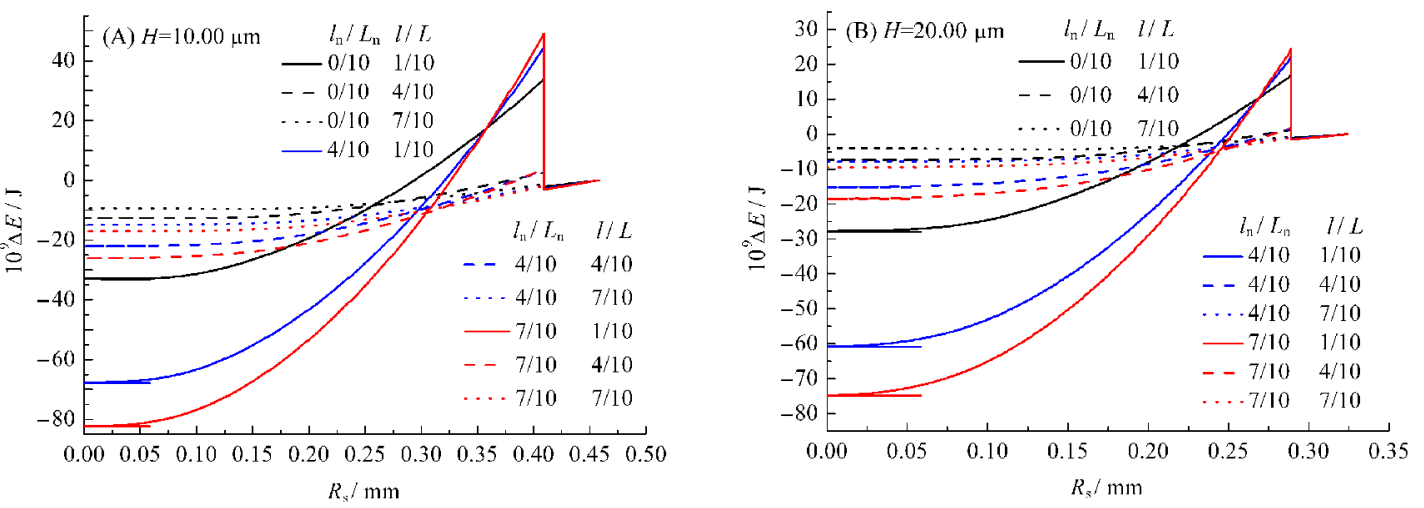

图 4 超疏水表面上冷凝液滴形变过程的能量变化

Fig.4 Energy changes of a condensed drop on SHS during its shape transformation $L=16.00 \mu \mathrm{m}, L_{\mathrm{n}}=16.00 \mathrm{~nm}, V=1.0 \mathrm{~nL}, \theta_{0}=110.0^{\circ}$

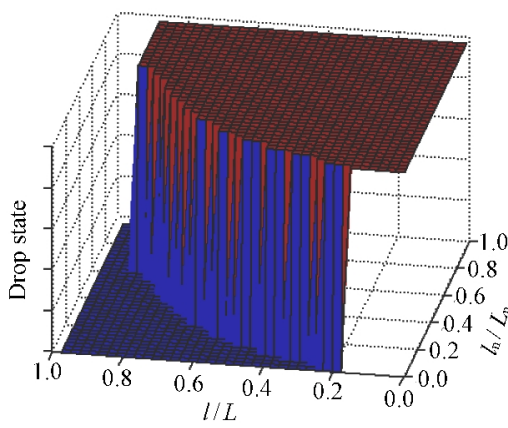

(A1)

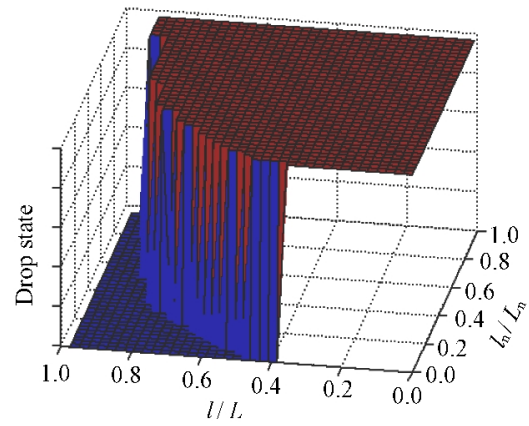

(A2)

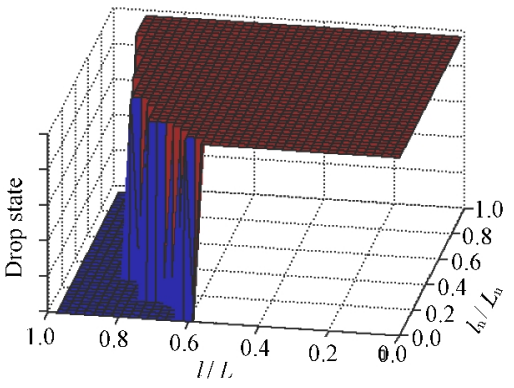

(A3)

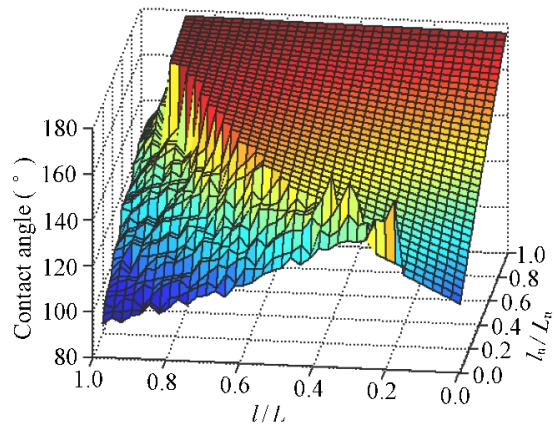

(B1)

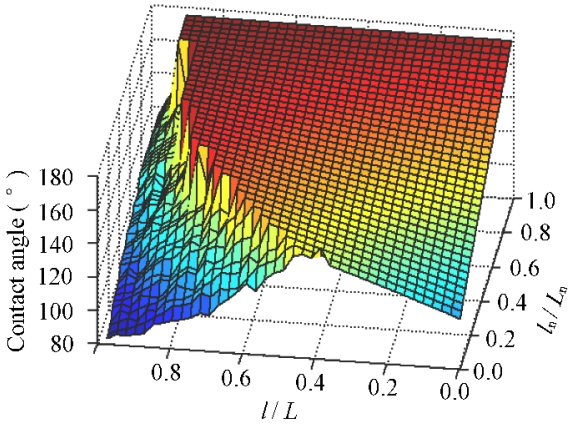

(B2)

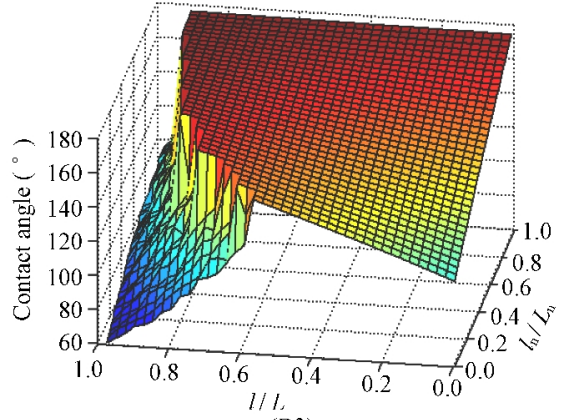

(B3)

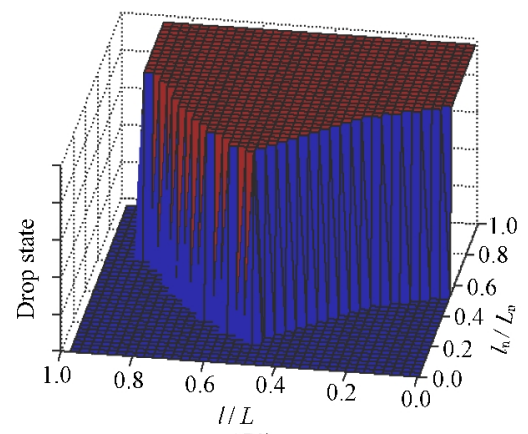

(C1)

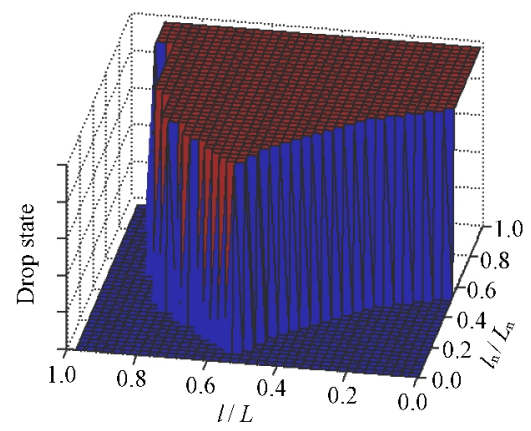

(C2)

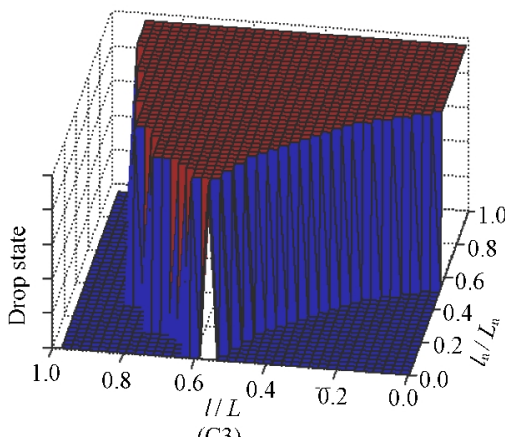

(C3)

图 5 超疏水表面上冷凝液滴最终状态与微纳结构尺度的关系

Fig.5 Effect of micro nano textures of SHS on final states of condensed drops

(A1)-(A3) drop states when $H$ is 10.00, 20.00 and $40.00 \mu \mathrm{m}$, respectively, above for Cassie state, below for Wenzel state;

(B1)-(B3) apparent $\theta$ when $H$ is $10.00,20.00$ and $40.00 \mu \mathrm{m}$, respectively; (C1)-(C3) drop states when $H$ is $10.00,20.00$ and $40.00 \mu \mathrm{m}$, respectively, above for Cassie state with $\theta \geqslant 150.0^{\circ}$, below for Wenzel or Cassie state with $\theta<150.0^{\circ} ; V=1.0 \mathrm{~nL}, \theta_{\mathrm{n}}=110.0^{\circ}$ 
表 1 超疏水表面上冷凝液滴状态的计算结果与文献实验结果的比较

Table 1 Comparison of calculated condensed drop states on SHS with experimental results

\begin{tabular}{|c|c|c|c|c|c|c|c|c|c|c|}
\hline \multicolumn{5}{|c|}{ Textured parameters } & \multicolumn{3}{|c|}{ Drop state in experiments } & \multicolumn{3}{|c|}{ Drop state calculated in this work } \\
\hline$H / \mu \mathrm{m}$ & $l / \mu \mathrm{m}$ & $L / \mu \mathrm{m}$ & $f_{\mathrm{n}}$ & $\theta_{0}$ & State & $\theta$ & Reference & State & $\theta$ & $V / n L$ \\
\hline 5.20 & 6.30 & 11.20 & 0.25 & $101.0^{\circ}$ & Cassie & unmeasured & [10] & Cassie & $164.0^{\circ}$ & $0.1-1.0$ \\
\hline 5.20 & 6.30 & 11.20 & 1.00 & $101.0^{\circ}$ & Wenzel & unmeasured & [10] & Wenzel & $94.0^{\circ}-100.9^{\circ}$ & $0.1-1.0$ \\
\hline 8.00 & 8.30 & 12.00 & 0.25 & $101.0^{\circ}$ & Cassie & unmeasured & [10] & Cassie & $168.8^{\circ}$ & $0.1-1.0$ \\
\hline 8.00 & 8.30 & 12.00 & 1.00 & $101.0^{\circ}$ & Wenzel & unmeasured & [10] & Wenzel & $84.3^{\circ}-98.6^{\circ}$ & $0.1-1.0$ \\
\hline- & - & - & $1.00 \times 10^{-4}$ & $117.0^{\circ}$ & Cassie & $179.4^{\circ}$ & [11] & Cassie & $179.5^{\circ}$ & \\
\hline- & - & - & 0.11 & $108.0^{\circ}$ & Cassie & $160^{\circ}-170^{\circ}$ & [12] & Cassie & $157.4^{\circ}$ & \\
\hline \multirow[t]{2}{*}{45.20} & 13.60 & 37.30 & 1.00 & $117.3^{\circ}$ & Wenzel+Cassie & $88.1^{\circ}-115.9^{\circ}$ & {$[7]$} & Wenzel $(1.0 \mathrm{~nL})+$ Cassie $(10.0 \mathrm{~nL})$ & $143.2^{\circ}$ & 1.0 \\
\hline & & & & & & & & & $141.4^{\circ}$ & 10.0 \\
\hline \multirow[t]{2}{*}{40.00} & 8.00 & 16.00 & 1.00 & $118.0^{\circ}$ & Wenzel+Cassie & Unmeasured & {$[6]$} & Wenzel $(0.1 \mathrm{~nL})+$ Cassie $(1.0 \mathrm{~nL})$ & $108.9^{\circ}$ & 0.1 \\
\hline & & & & & & & & & $150.2^{\circ}$ & 1.0 \\
\hline \multirow[t]{2}{*}{40.00} & 8.00 & 12.00 & 1.00 & $118.0^{\circ}$ & Wenzel+Cassie & Unmeasured & {$[6]$} & Wenzel $(0.1 \mathrm{~nL})+$ Cassie $(1.0 \mathrm{~nL})$ & $77.7^{\circ}$ & 0.1 \\
\hline & & & & & & & & & $160.2^{\circ}$ & 1.0 \\
\hline \multirow[t]{2}{*}{40.00} & 24.00 & 32.00 & 1.00 & $110.0^{\circ}$ & Wenzel & $24^{\circ}-120^{\circ}$ & {$[1]$} & Wenzel & $35.2^{\circ}$ & 0.1 \\
\hline & & & & & & & & & $102.6^{\circ}$ & 10.0 \\
\hline \multirow[t]{2}{*}{62.00} & 32.00 & 64.00 & 1.00 & $90.0^{\circ}$ & Wenzel & Unmeasured & [2] & Wenzel & $37.6^{\circ}$ & 1.0 \\
\hline & & & & & & & & & $61.1^{\circ}$ & 10.0 \\
\hline \multirow[t]{2}{*}{10.00} & 7.50 & 12.50 & 1.00 & $98.0^{\circ}$ & Wenzel & $129^{\circ}$ & {$[3]$} & Wenzel & $90.4^{\circ}$ & 1.0 \\
\hline & & & & & & & & & $101.3^{\circ}$ & 10.0 \\
\hline \multirow[t]{2}{*}{30.00} & 12.00 & 26.00 & 1.00 & $98.0^{\circ}$ & Wenzel & $126^{\circ}$ & [3] & Wenzel & $77.1^{\circ}$ & 1.0 \\
\hline & & & & & & & & & $94.0^{\circ}$ & 10.0 \\
\hline
\end{tabular}

Drop volumes in table were determined according to the sizes of condensed drops observed in that literature.

且接触角大的液滴下部的凸起肋个数都是最多的, 其实照片上也有很多接触角较小的液滴, 只是他们 没有测量而已. 事实上, 当液滴小到其下部只覆盖几 个凸起胁时, 液滴的接触角将对其所在的具体位置 非常敏感, 当液滴根部大部分位于凸起胁间时, 接触 角将会较小, 而根部大部分位于凸起肋之上时, 接触 角将会较大. 本文的计算模型只适用于液滴根部覆 盖的凸起肋数目较多的情况.

本计算没有考虑液滴变形过程中三相线经过 微米尺度凸起胁时界面自由能的微小波动 ${ }^{[14-17]}$. Yamamoto 等 ${ }^{[14]}$ 的计算结果表明, 该波动能量的数量 级为 $10^{-10} \mathrm{~J}$ 或更低, 而本文计算的液滴变化过程中 的界面能数量级在 $10^{-8} \mathrm{~J}$. 因此, 三相线经过凸起胁 引起的能量波动可以忽略不计, 因为微小的震动能 量或液滴变形的惯性就可以克服这个能量波动造成 的微小能垒. 而且当表面上有纳米二级粗糙结构以 后, 这种能量波动将会变得更小. 此外, 由于冷凝液 滴的体积非常小, 液滴的重力可以忽略不计, 因此本 计算也没有考虑液滴的重力势能.

另外, 本文的计算结果表明, 具有适宜微纳二 级粗粘结构的 SHS 上的冷凝液滴可以自发地从 Wenzel 状态变成 Cassie 状态. Zheng 等 ${ }^{[22]}$ 在原位观
测荷叶上的冷凝液滴行为时发现, Wenzel 态的冷凝 液滴会从乳突根部向上迁移形成 Cassie 状态, 他们 把这种迁移归结为荷叶乳突表面存在 “润湿梯度”, 即乳突根部更不润湿, 而乳突上部相对润湿, 从而造 成推动力. 但是乳突表面的这种 “润湿梯度”是否存 在尚未被证明. 本计算表明, 荷叶上具有的微纳二级 粗糙结构可以自发地使 Wenzel 状态的液滴向 Cassie 状态的液滴转变, 并不需要这种 “润湿梯度” 带来的推动力.

最后, 本文就 SHS 上理想的滴状冷凝过程进行 如下描述: 初始冷凝液核在微米粗䊁结构内到处都 形成, 液核长大后进行不断的合并直至冷凝液充满 微米粗粘面的部分区域形成冷凝液斑, 其体积一般 在 0.1-1.0 nL, 液斑进而开始收缩底半径向 Wenzel 状态变化并变成 Cassie 状态液滴, 最后 Cassie 状态 液滴滚落脱离壁面. 这个过程非常快, 并且表面上有 无数个这样的液滴形成和脱落, 从而大大强化冷凝 传热过程. 文献[23]为了实现Cassie 状态的冷凝液 滴, 采用控制凸起胁上端面较为润湿而凸起胁侧面 和底面不润湿的策略, 使得冷凝液滴只在凸起胁上 部形成. 但是这种方法只利用了粗粘表面的少部分 面积, 而没有充分利用所有的冷凝表面, 因此冷凝传 
热速率将受到抑制.

\section{4 结 论}

(1) 在具有微纳二级尺度的 SHS 上, 较大尺度 液滴的表观接触角仍服从 Cassie 或 Wenzel 公式, 只需将原来的本征接触角换成纳米结构表面上液滴 的表观接触角.

(2) SHS 上初始冷凝的微小液滴在微尺度结构 内部合并并充满微结构高度后, 将沿着液滴底半径 减小、液滴能量降低的方向变化, 当液滴能量不再降 低时就是液滴的最终稳定状态, 可能是 Wenzel 也 可以是 Cassie 状态, 取决于表面的微纳米结构特征.

（3）在只有微米尺度粗粘结构的表面上, 冷凝夜 滴很难形成接触角高于 $150^{\circ}$ 的 Cassie 状态, 只有当 表面具有微纳米二级粗粘结构, 且纳米结构的表面 空气面积分率较高时, 冷凝液滴才能成为超疏水状 态.

(4) SHS 上的冷凝液滴由于尺度较小, Wenzel 状态的液滴在其底部粗鉌结构内的液体体积已经不 可忽略, 因此, Wenzel 状态的冷凝液滴不再符合 Wenzel 方程.

(5) 表面的微纳米粗糙结构对于冷凝液滴的最 终状态、接触角、滚动和脱落等均具有重要影响, 需 要适宜设计 SHS 的微纳米二级粗糙结构, 才能实现 理想的滴状冷凝过程.

\section{References}

1 Wier, K. A.; McCarthy, T. J. Langmuir, 2006, 22(6): 2433

2 Narhe, R. D.; Beysens, D. A. Langmuir, 2007, 23(12): 6486

3 Jung, Y. C.; Bhushan, B. Journal of Microscopy, 2008, 229(1): 127

4 Narhe, R. D.; Beysens, D. A. Phys. Rev. Lett., 2004, 93(7): 076103

5 Narhe, R. D.; Beysens, D. A. Europhys. Lett., 2006, 75(1): 98
6 Dorrer, C.; Ruhe, J. Langmuir, 2007, 23(7): 3820

7 Chen, X. L.; Lu, T. Science in China Series G-Physics Mechanics and Astronomy, 2009, 52(2): 233

8 Song, Y. J.; Ren, X. G.; Ren, S. M.; Wang, H. Journal of Engineering Thermophysics, 2007, 28(1): 95 [宋永吉, 任晓光, 任绍梅, 王 虹. 工程热物理学报, 2007, 28(1): 95]

9 Chen, L.; Liang, S. Q.; Yan, R. S.; Cheng, Y. J.; Huai, X. L.; Chen, S. L. Journal of Thermal Science, 2009, 18(2): 160

10 Chen, C. H.; Cai, Q. J.; Tsai, C. L.; Chen, C. L.; Xiong, G. Y.; Yu, Y.; Ren, Z. F. Appl. Phys. Lett., 2007, 90(17): 173108

11 Dorrer, C.; Ruhe, J. Advanced Materials, 2008, 20(1): 159

12 Lau, K. K. S.; Bico, J.; Teo, K. B. K.; Chhowalla, M.; Amaratunga, G. A. J.; Milne, W. I.; McKinley, G. H.; Gleason, K. K. Nano Letters, 2003, 3(12): 1701

13 Barbieri, L.; Wagner, E.; Hoffmann, P. Langmuir, 2007, 23: 1723

14 Yamamoto, K.; Ogata, S. Journal of Colloid and Interface Science, 2008, 326(2): 471

15 Li, W.; Amirfazli, A. Journal of Colloid and Interface Science, 2005, 292(1): 195

16 Li, W.; Amirfazli, A. Advances in Colloid and Interface Science, 2007, 132(2): 51

17 Li, W.; Cui, X. S.; Fang, G. P. Langmuir, 2010, 26(5): 3194

18 Carbone, G.; Mangialardi, L. The European Physical Journal E, 2005, 16(1): 67

19 Werner, O.; Wagberg, L.; Lindstrom, T. Langmuir, 2005, 21(26): 12235

20 Patankar, N. A. Langmuir, 2004, 20(17): 7097

21 Patankar, N. A. Langmuir, 2004, 20(19): 8209

22 Zheng, Y. M.; Han, D.; Zhai, J.; Jiang, L. Appl. Phys. Lett., 2007, 92(8): 084106

23 Varanasi, K. K.; Hsu, M.; Bhate, N.; Yang, W. S.; Deng, T. Appl. Phys. Lett., 2009, 95(9): 094101 